\title{
Upregulated NOX1 expression in gallbladder cancer-associated fibroblasts predicts a poor prognosis
}

\author{
FANG-TAO WANG ${ }^{*}$, MOHAMED HASSAN ${ }^{*}$, KAMAR HASAN ANSARI* \\ GUO-LI XU, XIN-PING LI and YUE-ZU FAN
}

Department of Surgery, Tongji Hospital, Tongji University School of Medicine, Tongji University, Shanghai 200065, P.R. China

Received December 11, 2018; Accepted June 21, 2019

DOI: 10.3892/or.2019.7249

\begin{abstract}
Gallbladder cancer (GBC) is a lethal aggressive malignant neoplasm of the biliary tract. Potential prognostic markers and therapeutic targets for this disease are urgently required. Cancer-associated fibroblasts (CAFs) play a key role in tumorigenesis and the development of cancer. Nicotinamide adenine dinucleotide phosphate oxidase 1 (NOX1) expression has been reported to be involved in tumorigenesis and useful for tumor prognosis. However, NOX1 expression in the stroma of GBCs, particularly gallbladder cancer-associated fibroblasts (GCAFs), and its prognostic significance in GBC patients remains unclear. In the present study, NOX1 expression in the stroma of human gallbladder lesions in vivo was investigated, as well as in GCAFs and co-cultures of GBC-SD+GCAFs in vitro, and their correlation with clinicopathological parameters and the prognosis of GBC patients were evaluated. The results revealed that NOX1 expression was significantly upregulated in the stroma of GBCs compared with precancerous
\end{abstract}

Correspondence to: Professor Yue-Zu Fan, Department of Surgery, Tongji Hospital, Tongji University School of Medicine, Tongji University, 389 Xincun Road, Shanghai 200065, P.R. China E-mail: fanyuezu@hotmail.com

*Contributed equally

Abbreviations: GBC, gallbladder cancer; CAFs, cancer-associated fibroblasts; GCAFs, gallbladder cancer-associated fibroblasts; NFs, normal fibroblasts; NOX, nicotinamide adenine dinucleotide phosphate oxidase; TME, tumor microenvironment; ROS, reactive oxygen species; GBPL, gallbladder precancerous lesion; GBBL, gallbladder benign lesion; OS, overall survival; IHC, immunohistochemistry; DAB, 3,3-diaminobenzidine; PBS, phosphate-buffered saline; MOD, mean optical density; IOD, integrated optical density; $\alpha$-SMA, $\alpha$-smooth muscle actin; FSP-1, fibroblast secreted protein-1; FAP, fibroblast activation protein; CIF, co-immunofluorescence; FBS, fetal bovine serum; DAPI, diamidine phenylindole; DMEM, Dulbecco's modified Eagle's media; RT-qPCR, reverse transcription-quantitative polymerase chain reaction; FC, fold change; GO, gene ontology; IVT, in vitro transcription

Key words: gallbladder neoplasm, cancer-associated fibroblast, NOX1, prognosis and benign lesions of the gallbladder; NOX1 expression was localized to gallbladder stromal fibroblasts expressing $\alpha$-smooth muscle actin and fibroblast secreted protein-1. Furthermore, these observations were confirmed by the fact that NOX1 expression was upregulated in GCAFs as determined by Affymetrix gene profile chip analysis and reverse transcription-quantitative PCR. In addition, overexpression was observed in formed spheroids of GBC-SD+GCAF co-cultures by immunohistochemistry and western blotting in vitro. Thus, it was verified that NOX1 expression was upregulated in GCAFs. Furthermore, upregulated stromal NOX1 expression was correlated with aggressive characteristics such as differentiation degree $(\mathrm{P}=0.042)$, venous invasion $(\mathrm{P}=0.041)$, resection methods $(\mathrm{P}=0.002)$, and a lower survival rate $(\mathrm{P}=0.025, \log$-rank test $)$ of patients with GBC. Stromal NOX1 expression $(\mathrm{P}=0.047)$ was an independent prognostic factor for the overall survival rate of patients with GBC. GBC patients with upregulated NOX1 expression in GCAFs had a poorer prognosis. These results revealed that stromal NOX1 may be a novel biomarker and/or target, and may contribute to the discovery of new tumor markers and potential targeted therapeutics for human GBCs.

\section{Introduction}

Gallbladder cancer (GBC), a lethal aggressive malignant neoplasm, is the most common malignancy of the biliary tract and the leading cause of cancer-related mortalities in western countries and China (1-5). The majority of patients with GBC are diagnosed at an advanced stage due to early occult symptoms, and treating GBC is associated with many problems, not only due to the poor results of surgical resection for the disease, but also due to its insensitivity to chemo-radiotherapy; thus, the prognosis of the patients is still very poor (5-7). Therefore, it is necessary to explore the prognostic indicators and drug targets of GBC, and provide more effective and individualized treatments for patients with GBC.

Cancer-associated fibroblasts (CAFs) are key cellular components in tumor stroma and the most primary stromal cells of the tumor microenvironment (TME). Recent studies have demonstrated the critical role of CAFs in cancer stroma for tumorigenesis, development and the targeted therapy of cancers (8-10). In addition, tumor treatment effectiveness is complicated by the presence of reactive stroma in the 
TME, which is associated with tumor invasiveness and drug resistance (10-13). CAFs have their own gene expression profiles that are different from normal fibroblasts (NFs), and they interact with cancer cells via a variety of signals in a paracrine or autocrine manner to affect the TME, determine cancer cell growth, invasion, metastasis, angiogenesis and therapeutic tolerance (14-17), and predict the poor prognosis of patients $(18,19)$. In addition, anti-CAFs can effectively prevent tumor progression before tumor invasion. In pancreatic cancer, as well as in other cancer treatments, inhibiting CAFs can prolong the survival of patients compared with chemotherapy alone (20-23). However, little is known about the relationship between the complex components of CAFs and the prognosis of patients with tumors, and there is a lack of studies on related molecular mechanisms. In order to predict the poor prognosis of patients and to tailor treatments more effectively to the individual patient, it is important to clearly define the tumor stroma, particularly CAFs, at a molecular level, which will enable researchers to identify biomarkers that will more accurately predict patient prognosis and responsiveness to treatments.

Nicotinamide adenine dinucleotide phosphate (NADPH) oxidase 1 (NOX1) is a member of the NADPH oxidase family. The main biological function of NOX1 and other NOX family proteins is to produce reactive oxygen species (ROS) (24). ROS are oxygen-derived small molecules that are oxidizing factors and can be easily converted into free radicals $(25,26)$. NOX-dependent ROS regulation abnormalities are thought to be closely associated with tumor development $(27,28)$. NOX1 has been reported to be highly expressed in a variety of tumor types including gastric (29-31) and liver cancer (32), and is associated with poor prognosis. However, tumor stromal NOX1 expression and its relationship with the prognosis of tumor patients have not been reported. In the present study, the relationship between NOX1 expression in GCAFs and the prognosis of GBC patients was investigated. The results revealed that NOX1 expression was significantly upregulated in the stroma of GBCs, and this observation was confirmed by GBC specimens expressing the interstitial marker $\alpha$-smooth muscle actin (SMA) and fibroblast secreted protein (FSP)-1 positive in vivo and by GCAF and GBC-SD+GCAF co-culture at the mRNA and protein levels in vitro. GBC patients with upregulated stromal NOX1 expression had a lower survival rate. Thus, it was concluded that upregulated NOX1 expression in GCAFs may predict an unfavorable prognosis. This may contribute to the discovery of novel tumor markers and potential targeted therapeutics for human GBCs.

\section{Materials and methods}

Patients and clinical specimens. The present study was conducted in accordance with ethical standards, the Declaration of Helsinki and the official recommendations of the Chinese Community Guidelines, and was approved by the Ethics Committee and the Institutional Review Board of Tongji Hospital. Written informed consent was obtained from all patients. A total of 81 cases of paraffin-embedded gallbladder tissue specimens, including 65 GBC specimens, 8 gallbladder precancerous lesion (GBPL; adenoma and severe dysplasia) specimens and 8 gallbladder benign lesion (GBBL; cholecystitis) specimens were collected. All GBC patients underwent surgery from January 2007 to September 2012 at Tongji Hospital, Tongji University School of Medicine, were histopathologically diagnosed and did not receive chemotherapy or radiotherapy before surgery. To avoid the direct impact of surgery, patients who died within a month after surgical resection were excluded. Two independent pathologists who were unaware of the clinical status of patients validated the diagnosis of these GBC samples. Detailed clinicopathological and follow-up data were collected from the medical records of patients at Tongji Hospital, and completed by telephone survey. The median follow-up time was 19.9 (range, 1-62) months for all GBC patients. The 5-year overall survival (OS) rate was $12.3 \%$ (8/65). The demographic and clinicopathological data for a total of 65 GBC patients are summarized in Table I.

Immunohistochemistry (IHC) in vivo. IHC staining was used to detect the expression of NOX1 protein in the stroma of different gallbladder tissue specimens. After deparaffinization and inactivating endogenous peroxide, sections $(4 \mu \mathrm{m})$ were pretreated using bovine serum albumin $\mathrm{V}$ working solution (cat. no. A8020; Beijing Solarbio Science \& Technology Co., Ltd.), and then incubated with the primary anti-rabbit antibody against NOX1 (1:500; cat. no. GTX 103888; GeneTech), followed by the secondary antibody immunoglobulin (Ig; H+L; 1:200; cat. no. 074-1506; KPL) and DAB (cat. no. K346711; Dako; Agilent Technologies, Inc.) solution, and were counterstained with hematoxylin according to the manufacturer's instructions. A negative control was conducted by replacing the primary antibody with PBS (Gibco; Thermo Fisher Scientific, Inc.) in all samples. Known immunoassay-positive colon cancer sections were used as the positive controls.

The positive expression of NOX1 protein was localized in the cytoplasm, nucleus and stroma. In the present study, only the expression of NOX1 was observed in the stroma of gallbladder tissues. To analyze biomarker expression in stromal tissues, the present study modified the methods described in previous studies (33-35). The staining of NOX1 in cancer tissues was evaluated in five randomly selected high-power fields, which were considered to represent the average value of a tumor at x200 magnification. Images were first imported into Adobe Photoshop. Hue and saturation of the images were normalized using Auto-Contrast. Tumor epithelium was distinguished from the stroma by differences in nuclear and cellular morphology, and tissue architecture. Using the lasso tool, epithelial tissues were selected and cropped out from the image, leaving the stromal tissues behind. These stromal tissues were labeled as 'total stromal area'. DAB chromogen staining (brown) was selected using the Magic Wand Tool in the Color Range Window. The selected pixels were copied and pasted into a new window and saved as a separate file. Then, NOX1 positive staining in 'total stromal area' was scored using mean optical density (MOD). The MOD formula was as follows: Integrated optical density/(960x1,280, pixel value). Based on the results of stroma positive staining, a MOD value of 0.138 was used to distinguish low (MOD <0.138) and high (MOD $\geq 0.138$ ) NOX1 expression. Two independent researchers blinded to the clinicopathological parameters and patient outcome scored all gallbladder tissue samples. Experienced pathologists assessed any inconsistencies. 
Table I. Demographic and clinicopathological parameters of GBC patients included in the present study.

\begin{tabular}{|c|c|}
\hline $\begin{array}{l}\text { Demographic and } \\
\text { clinicopathological parameters }\end{array}$ & $\begin{array}{l}\text { Patients witl } \\
\text { GBC [n (\%) }\end{array}$ \\
\hline No. of patients & 65 \\
\hline \multicolumn{2}{|l|}{ Sex } \\
\hline Male & $25(38.5)$ \\
\hline Female & $40(61.5)$ \\
\hline \multicolumn{2}{|l|}{ Age (years) } \\
\hline$>65$ & $34(52.3)$ \\
\hline$\leq 65$ & $31(47.7)$ \\
\hline \multicolumn{2}{|l|}{ Tumor size $(\mathrm{cm})$} \\
\hline$>3$ & $27(41.5)$ \\
\hline$\leq 3$ & $38(58.5)$ \\
\hline \multicolumn{2}{|l|}{ Tumor location } \\
\hline Bottom & $26(40)$ \\
\hline Corporis and others & $39(60)$ \\
\hline \multicolumn{2}{|l|}{ Pathological diagnosis } \\
\hline Adenocarcinoma & $61(93.8)$ \\
\hline Others $^{\mathrm{a}}$ & $4(6.2)$ \\
\hline \multicolumn{2}{|l|}{ Differentiation degree } \\
\hline G1 (high) & $11(16.9)$ \\
\hline G2 (moderate) & $29(44.6)$ \\
\hline G3 (poor) & $25(38.5)$ \\
\hline \multicolumn{2}{|l|}{ Nevin stage } \\
\hline $\mathrm{S} 1-\mathrm{S} 2$ & $8(12.3)$ \\
\hline S3-S5 & $57(87.7)$ \\
\hline \multicolumn{2}{|l|}{ Lymph node metastasis } \\
\hline$(-)$ & $21(32.3)$ \\
\hline$(+)$ & $44(67.7)$ \\
\hline \multicolumn{2}{|l|}{ Liver infiltration } \\
\hline$(-)$ & $34(52.3)$ \\
\hline$(+)$ & $31(47.7)$ \\
\hline \multicolumn{2}{|l|}{ Venous invasion } \\
\hline$(-)$ & $36(55.4)$ \\
\hline$(+)$ & $29(44.6)$ \\
\hline \multicolumn{2}{|l|}{ Curability } \\
\hline $\mathrm{R} 0$ & $32(49.2)$ \\
\hline $\mathrm{R} 1, \mathrm{R} 2$ & $33(50.8)$ \\
\hline
\end{tabular}

${ }^{a}$ Mucinous adenocarcinoma, squamous cell carcinoma, adenosquamous carcinoma. GBC, gallbladder cancer.

Co-immunofluorescence $(C I F)$ in vivo. CIF staining was used to verify the expression of NOX1 protein in the stroma of GBC specimens using stromal markers such as $\alpha$-SMA and FSP-1. After deparaffinizing and pretreating specimens as aforementioned for IHC, sections $(4 \mu \mathrm{m})$ from GBC tissues were permeabilized in PBS containing 10\% methanol for $30 \mathrm{~min}$, washed in PBS and blocked for $1 \mathrm{~h}$ with PBS containing 3\% FBS (Hangzhou Sijiqing Biological Engineering Materials Co., Ltd.). Mouse IgG was blocked using the M.O.M kit (cat. no. BMK-2202; Vector Laboratories, Inc.) according to the manufacturer's protocol. For CIF staining of NOX1 and $\alpha$-SMA, or NOX1 and FSP-1, sections were respectively incubated with rabbit anti-NOX1 (1:500; cat. no. gtx103888; GeneTex) and mouse anti- $\alpha$-SMA (1:200; cat. no. ab7817; Abcam), or rabbit anti-NOX1 (1:500) and mouse anti-FSP-1 (1:100; cat. no. ab93283; Abcam) at $4^{\circ} \mathrm{C}$ overnight; then incubated with the corresponding secondary antibody, goat anti-rabbit IgG (1:1,000; cat. no. ab6717; Abcam) to detect NOX1 expression, goat anti-mouse IgG (1:200; cat. no. A32727; Thermo Fisher Scientific, Inc.) to detect $\alpha$-SMA or FSP-1 expression. Finally, sections were stained with DAPI for $5 \mathrm{~min}$, and observed at x200 magnification using an immunofluorescence microscopy.

Cell lines and cultures. The human gallbladder cancer cell line GBC-SD (Shanghai Cell Biology Research Institute of Chinese Academy of Sciences) was maintained and propagated in DMEM (Gibco; Thermo Fisher Scientific, Inc.) supplemented with $10 \%$ FBS and $10^{5} \mathrm{U} \cdot \mathrm{ml}^{-1}$ penicillin and streptomycin (Shanghai Pharmaceutical Works) in an incubator (Forma series II HEPA Class 100; Thermo Fisher Scientific, Inc.) at $37^{\circ} \mathrm{C}$ with a $5 \% \mathrm{CO}_{2}$ atmosphere. Human GCAFs and NFs were isolated from the clinical specimens of human GBC tissues and adjacent normal tissues, and identified by the detection of the stromal markers $\alpha$-SMA and fibroblast activation protein (FAP) using IHC, immunofluorescence and western blotting. Established GCAFs and NFs were incubated in DMEM/F-12 medium (Gibco; Thermo Fisher Scientific, Inc.) supplemented with $10 \%$ FBS in an incubator (Thermo Fisher Scientific, Inc.) at $37^{\circ} \mathrm{C}$ with a $5 \% \mathrm{CO}_{2}$ atmosphere. The cells used in the experiment were between the 4th and 9 th generations.

Co-cultures of GBC-SD cells and GCAFs or NFs were performed by coating 96-well U-bottom plates (cat. no. 3799; Corning Incorporated) with poly-2-hydroxyethyl methacrylate (poly-Hema; Polysciences Europe GmbH). In poly-Hema coated 96-well plates, $2.5 \times 10^{5}$ GBC-SD cells were seeded per well as the mono-culture, and $1 \times 10^{5} \mathrm{GBC}-\mathrm{SD}$ cells and $1.5 \times 10^{5}$ GCAFs or NFs per well for co-cultures. Monocultures and co-cultures were incubated for 7 days in an incubator at $37^{\circ} \mathrm{C}$ with a $5 \% \mathrm{CO}_{2}$ atmosphere until spheroid formation.

Affymetrix chip analysis of gene expression profile and reverse transcription-quantitative (RT-q)PCR in GCAFs/NFs in vitro. To further verify the expression of NOX1 protein in the stroma of GBCs, especially in CAFs, the present study analyzed the gene expression profile in GCAFs/NFs using Affymetrix chip analysis and detected the different expression levels of NOX1 at the mRNA level in GCAFs/NFs using RT-qPCR in vitro.

Affymetrix chip analysis in vitro was performed using Affymetrix GeneChip Human 1.0ST array (Affymetrix; Thermo Fisher Scientific, Inc.). Briefly, total RNA was extracted in triplicate from GCAFs/NFs. After total RNA quality detection, RNA RT and in vitro transcription (IVT) of cRNA were performed by adding $130 \mu \mathrm{l}$ of the IVT Master Mix using a GeneChip 3'IVT PLUS Kit (Affymetrix; Thermo Fisher Scientific, Inc.) to $130 \mu 1$ of double-stranded cRNA. The generated cRNA was then synthesized, purified and labeled. Finally, after being hybridized and washed using a GeneChip Hybridization Wash and Stain kit (Affymetrix; Thermo Fisher Scientific, Inc.), arrays were scanned for differentially 
expressed genes between GCAFs/NFs using a Genechip Array scanner 3000 (Affymetrix; Thermo Fisher Scientific, Inc.). Array data were normalized using log scale robust multi-array analysis and were analyzed by R-Project software. Gene expression was deemed significant if the fold change (FC) value was $>1.5$ or $<0.67$, and $\mathrm{P}<0.05$. Gene Ontology (GO) analysis was used to perform functional enrichment analysis. For statistical analysis of GO, gene set enrichment analysis and Fisher exact analysis were performed. For the study of gene expression profile variance between GCAFs and NFs, potentially related up- or downregulated genes involved in biological processes were selected for verification.

For RT-qPCR, total RNA from GCAFs or NFs was prepared using TRIzol reagent (Invitrogen; Thermo Fisher Scientific, Inc.). The concentration of RNA was determined by absorption at 260-280 nm. PCR amplifications were performed with gene-specific primers with annealing temperatures and the number of amplification cycles optimized using cDNA in each group. The primers for NOX1 and GAPDH were as follows: NOX1, 5'-ACCTCTTGACAATGGGAAAC-3' (sense) and 5'-CTCCACTGTCGTGTTTCG-3' (antisense); and GAPDH, 5'-CTCCTCCTGTTCGACAGTCA-3' (sense) and 5'-GCT CCGCCCAGATACCATT-3' (antisense). PCR amplification reactions were performed as follows: $94^{\circ} \mathrm{C}$ for $3 \mathrm{~min}$, followed by 40 cycles of $95^{\circ} \mathrm{C}$ for $15 \mathrm{sec}, 60^{\circ} \mathrm{C}$ for $30 \mathrm{sec}, 72^{\circ} \mathrm{C}$ for $30 \mathrm{sec}$, and $82-86^{\circ} \mathrm{C}$ (fluorescence collection) for 5-10 sec, and finally $72-99^{\circ} \mathrm{C}$ for $5 \mathrm{~min}$. GAPDH primers were used as the control for PCR amplification. PCR products $(10 \mu \mathrm{l})$ were placed onto $15 \mathrm{~g} \cdot \mathrm{l}-1$ agarose gels and observed by ethidium bromide (Huamei Bioengineering Co., Ltd.) staining using ABI Prism 7300 SDS software (Bio-Rad Laboratories, Hercules, CA, USA). Data were analyzed using the $\Delta \Delta \mathrm{cq}$ method (36).

$I H C$ and western blotting in co-cultures of GBC-SD cells/GCAFs or NFs in vitro. To verify the elevated NOX1 expression in GCAFs, the present study further detected the altered expression of NOX1 at the protein level in co-cultures of GBC-SD cells/GCAFs or NFs using IHC and western blotting in vitro.

Co-culture spheroids of one 96-well plate were harvested, washed once in PBS and fixed in 4\% paraformaldehyde in PBS for $1 \mathrm{~h}$ at room temperature. After spheroid embedding in $1 \%$ agarose with PBS and a dehydration series, spheroids were embedded in paraffin. Sections $(1.5 \mu \mathrm{m})$ were incubated with primary anti-rabbit antibody against NOX1, then the secondary antibody $\operatorname{Ig}(\mathrm{H}+\mathrm{L})$ and finally with DAB solution according to the aforementioned steps used for IHC, and observed under an optical microscope (Olympus CH-2; Olympus Corporation). Negative controls were established by replacing the primary antibody with PBS in all samples. Ten sample slides (10 visual fields per slide) in each group were selected for analysis.

For western blotting, co-culture spheroids of one 96-well plate were harvested and washed once in PBS. Cells were lysed, the supernatant was recovered and BCA protein was determined with a protein quantitative kit (KangChen KC-430; Kangchen BioTech, Co., Ltd.). Then, an aliquot of $20 \mu \mathrm{g}$ of proteins was subjected to SDS-PAGE (10\%) under reducing conditions, and proteins were transferred to a PVDF membrane. The membrane was incubated with the NOX1 primary anti-rabbit antibody (1:3,000; cat. no. GTX 103888; GeneTech) and mouse anti-human GAPDH antibody (1:10,000; cat. no. KC-5G4; KangChen Biotech), followed by an appropriate anti-mouse horseradish peroxidase-labeled secondary antibody (1:5,000; cat. no. KC-MM-035; Kangchen BioTech Co., Ltd.). The target proteins were visualized using an enhanced chemiluminescent reagent ( $\mathrm{KC}^{\mathrm{TM}}$ Chemiluminescent kit; Kangchen BioTech Co., Ltd.), and imaged on a Bio-Rad chemiluminescence imager. The gray value and gray coefficient ratio of every protein were analyzed and calculated with ImageJ $1.37 \mathrm{v}$ analysis software (National Institutes of Health).

Statistical analysis. All statistical analyses were conducted using SPSS 22.0 software (IBM Corp.). Data are expressed as the mean \pm standard deviation (SD). Student's t-test was used to analyze independent samples. The $\chi^{2}$ test was used to analyze the relationship between NOX1 expression and clinicopathological characteristics. The Kaplan-Meier method and the log-rank test were used for survival analysis. Single variables and multivariate analysis of prognostic factors were performed using the Cox's regression model. $\mathrm{P}<0.05$ was considered to indicate a statistically significant difference.

\section{Results}

Expression of NOX1 protein is upregulated in GBC stroma with positive $\alpha$-SMA and FSP-1 expression, and elevated NOX1 expression in the stroma of GBC predicts poor prognosis. To determine the significance of NOX1 expression in the stroma of GBCs, the present study analyzed the expression of NOX1 at the protein level in the stroma of GBCs. Using IHC, the present study first analyzed the expression of NOX1 protein in different types of gallbladder tissues from GBBLs, GBPLs and GBCs. As revealed in Fig. 1, NOX1 protein was positively expressed (brown staining) in the tumor epithelium and in the stroma of different gallbladder tissues (Fig. 1A). The MOD value of NOX1 in the stroma of GBCs was significantly higher than that observed in the stroma of GBPLs $(0.138 \pm 0.030$ vs. $0.110 \pm 0.025, \mathrm{P}=0.028)$ or GBBLs $(0.138 \pm 0.030$ vs. $0.109 \pm 0.019, P=0.021)$; the MOD value of NOX1 in the GBPL stroma was higher than in the GBBL stroma, but the difference was not significant $(0.110 \pm 0.025$ vs. $0.109 \pm 0.019, \mathrm{P}=0.997$; Fig. 1B). Overall, these results indicated that NOX1 expression was upregulated in the stroma of GBCs compared with the stroma of GBPLs and GBBLs. Furthermore, to determine whether NOX1 protein was expressed in the stroma of GBCs, the present study performed CIF staining for the expression of NOX1 in GBC stroma tissues with $\alpha$-SMA and FSP-1. It was observed that the expression of NOX1 protein was positive not only in the tumor epithelium but also in the stroma of GBCs, consistent with DAB expression patterns. In addition, NOX1 overlapped with both $\alpha$-SMA and FSP-1-expressing cells, and co-localized with $\alpha$-SMA and FSP-1 positive stroma (Fig. 2), presenting NOX1 expression in both $\alpha$-SMA and FSP-1-positive fibroblasts in the stroma of GBCs. Thus, it is considered that the expression of NOX1 protein is upregulated in the stroma of GBCs.

To determine the significance of NOX1 expression in the stroma of GBCs, the present study used a MOD value of 0.138 to distinguish low (MOD $<0.138)$ and high $(\mathrm{MOD} \geq 0.138$ ) NOX1 expression, based on the results of the positive staining 
A
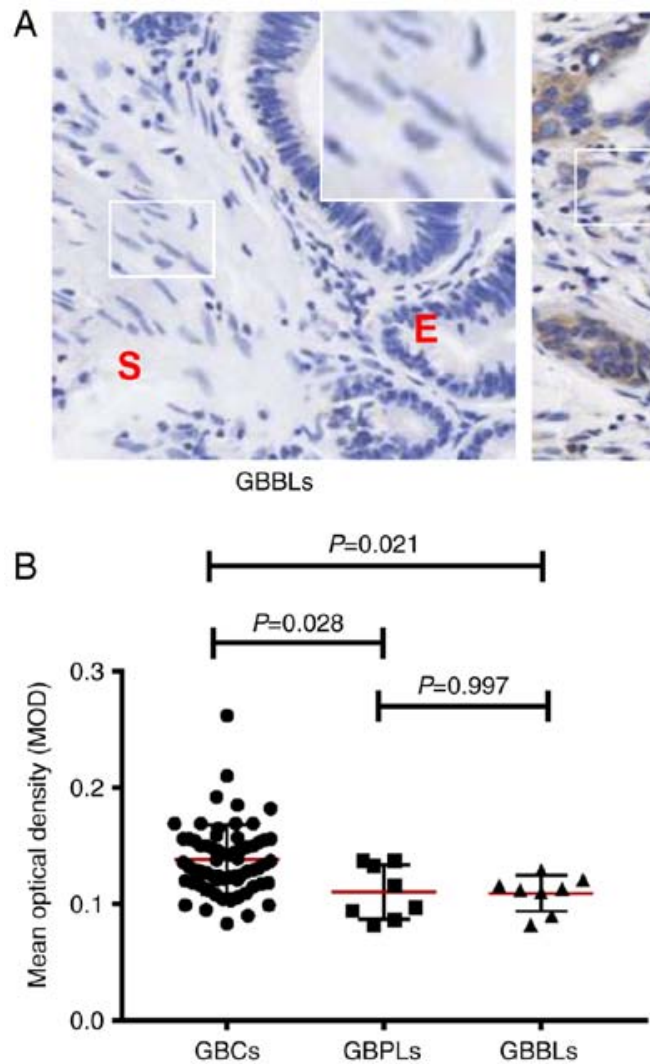

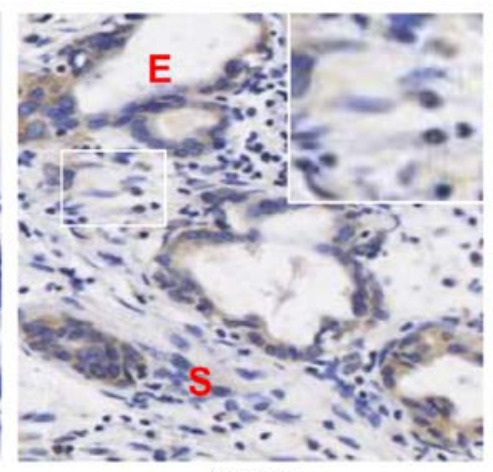

GBPLs

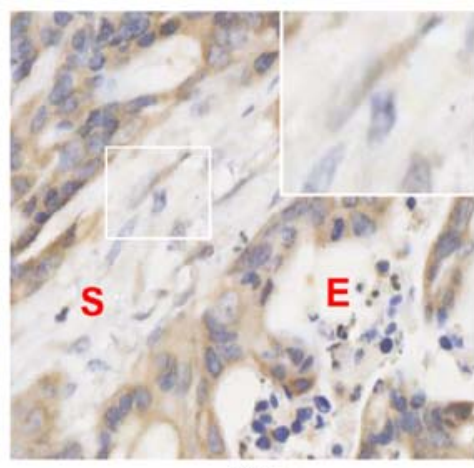

GBCs
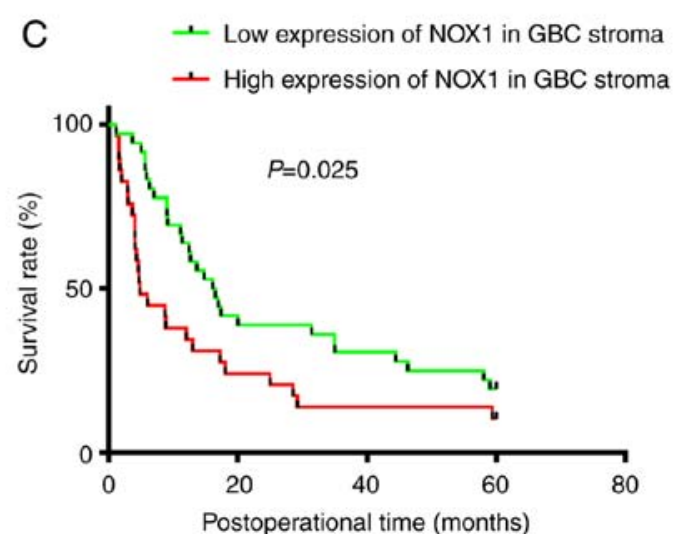

Figure 1. NOX1 expression is upregulated in the stroma of GBCs and Kaplan-Meier survival curves for the GBC patients with high and low stromal NOX1 expression. (A) Magnified images show representative NOX1 staining in the stroma (immunohistochemistry; magnification, x200). (B) Staining in the stroma was scored using MOD. Values are expressed as the mean \pm standard deviation. The expression of NOX1 protein in the stroma of GBCs ( $=65)$ was significantly upregulated compared with the stroma of GBBLs ( $\mathrm{n}=8 ; 0.138 \pm 0.030$ vs. $0.109 \pm 0.019, \mathrm{P}=0.021)$ or $\mathrm{GBPLs}(\mathrm{n}=8 ; 0.138 \pm 0.030$ vs. $0.110 \pm 0.025, \mathrm{P}=0.028)$; however, the difference of NOX1 expression in the stroma of both GBPLs and GBBLs was not significant $(0.110 \pm 0.025$ vs. 0.109 \pm 0.019 , $\mathrm{P}=0.997)$. $(\mathrm{C}) \mathrm{GBC}$ patients with upregulated stromal NOX1 expression had a lower survival rate than downregulated stromal NOX1 expression patients (P=0.025, log-rank test). E, epithelium; S, stroma; MOD, mean optical density; NOX1, nicotinamide adenine dinucleotide phosphate oxidase 1; GBPL, gallbladder precancerous lesions; GBBLs, gallbladder benign lesions; GBC, gallbladder cancers.

of the stroma. It was observed that stromal NOX1 was highly expressed in 29 cases (44.6\%) and poorly expressed in 36 cases (55.4\%) of GBCs. Upregulated NOX1 expression in the stroma of GBCs was significantly correlated with differentiation degree $(\mathrm{P}=0.042)$, venous invasion $(\mathrm{P}=0.041)$ and resection methods $(\mathrm{P}=0.002)$; but no significant correlations were observed between stromal NOX1 expression and the clinicopathological variables such as sex, age, tumor size, tumor location, histological type, Nevin stage, lymph node metastasis and liver infiltration (all $\mathrm{P}>0.05$; Table II). In addition, the present study used the Cox proportional hazards model to identify prognostic factors involved in GBC patients (Table III). Univariate analysis indicated that tumor histological type $(\mathrm{P}=0.011)$, differentiation degree $(\mathrm{P}=0.002)$, Nevin staging $(\mathrm{P}=0.012)$, lymph node metastasis $(\mathrm{P}=0.001)$, liver infiltration $(\mathrm{P}=0.002)$, vascular invasion $(\mathrm{P}<0.00001)$, curability $(\mathrm{P}<0.00001)$ and stromal NOX1 expression $(\mathrm{P}=0.027)$ were significantly associated with the OS of GBC patients. Multivariate analysis validated that histological type [hazard ratio (HR), 0.308; 95\% confidence interval $(\mathrm{CI}), 0.105-0.902 ; \mathrm{P}=0.032]$, differentiation degree (HR, 0.038; 95\% CI, 0.151-0.974; $\mathrm{P}=0.044)$, vascular invasion (HR, 2.375; 95\% CI, 1.363-4.139; $\mathrm{P}=0.002)$, curability (HR, 1.833; 95\% CI, 1.010-3.325; $\mathrm{P}=0.046)$ and stromal NOX1 expression (HR, 1.745; 95\% CI, 1.001-2.658; $\mathrm{P}=0.047)$ were the independent prognostic factors for the
OS rate of GBC patients. Furthermore, the log-rank test was used to evaluate the effect of stromal NOX1 expression on the survival of GBC patients. For 65 GBC patients enrolled in the present study, the mean and median survival time of the high-stromal NOX1 expression group (29/65, 44.6\%) were 15.7 and 4.8 months, respectively, with a 5-year survival rate of $10.3 \%$ (3/29), compared with 26.6 and 16.1 months, respectively, and a 5-year survival rate of $19.4 \%(7 / 36)$ for the low-stromal NOX1 expression group $(36 / 65,55.4 \%)$. The survival rate of GBC patients with upregulated stromal NOX1 expression was significantly lower than that of those with downregulated stromal NOX1 expression (Fig. 1C; $\mathrm{P}=0.025$, log-rank test). These results revealed that GBC patients with high stromal NOX1 expression have poorer prognoses.

NOX1 expression is upregulated in GCAFs. To verify the upregulated NOX1 expression in the stroma of GBCs, especially GCAFs, the present study performed Affymetrix chip analysis on the gene expression profile for GCAFs and NFs using the Affymetrix GeneChip Human 1.0ST array, and detected the expression of NOX1 at the mRNA level in GCAFs and NFs using RT-qPCR in vitro. As presented in Fig. 3, the associated volcano-map provided an overview of the significantly affected genes (Fig. 3A); GO analysis indicated the upregulated expression genes, based on the 


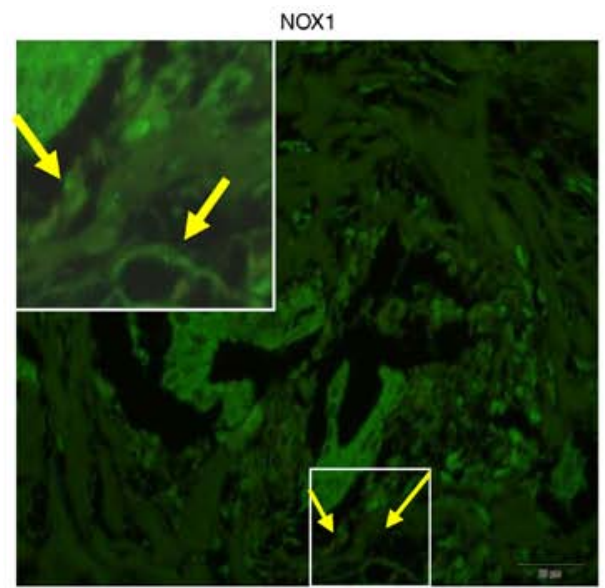

NOX1/FSP1

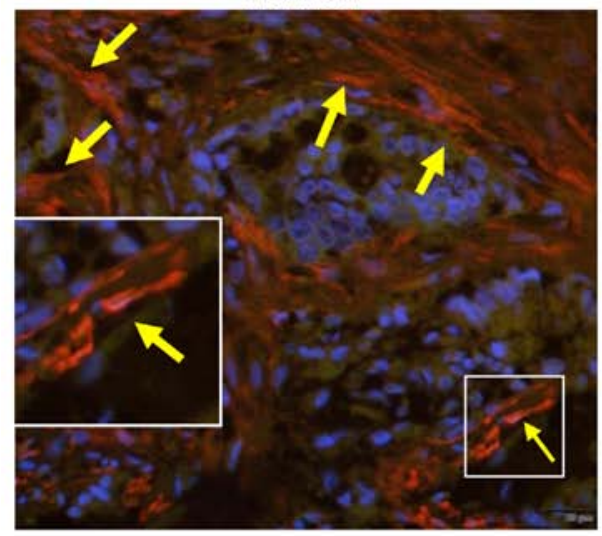

Anti-mouse-ThermoFisher-A32727

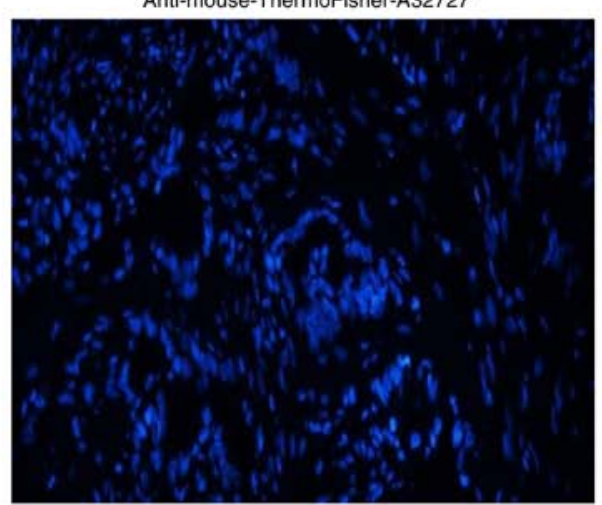

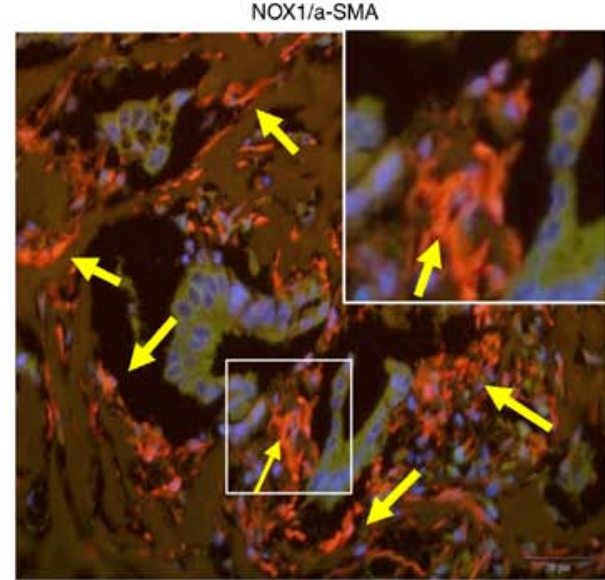

Anti-rabbit-Abcam-ab6717

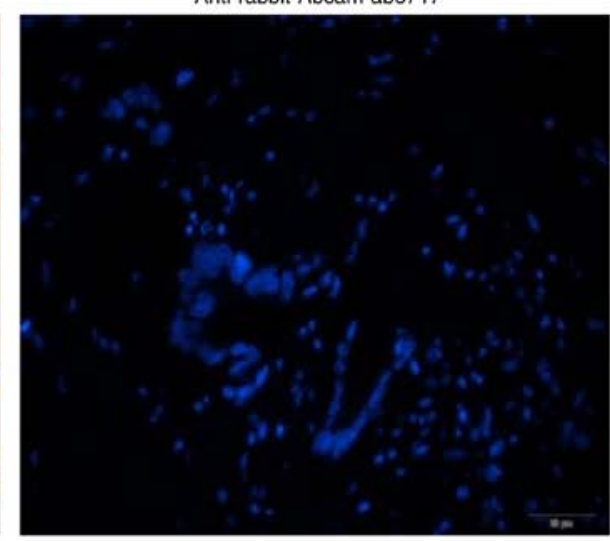

Anti-mouse-ThermoFisher-A32727

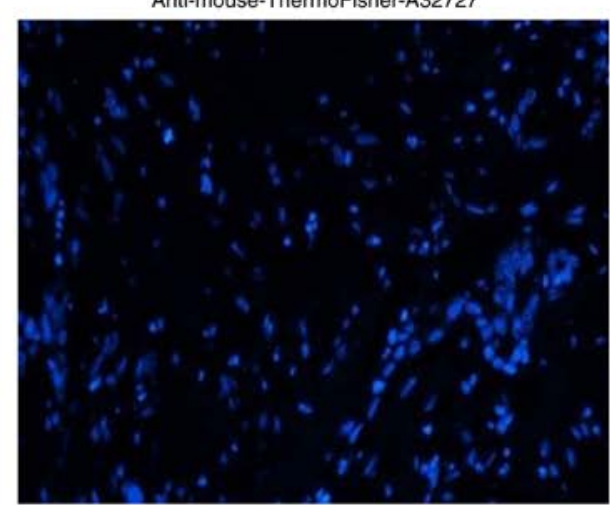

Figure 2. NOX1 is positively expressed in the stroma of GBCs with $\alpha$-SMA and FSP-1 positive expression. The expression of NOX1 (green) and $\alpha$-SMA or FSP-1 (red) in GBC tissues stained using co-immunofluorescence staining (immunofluorescence microscopy; magnification, x200). Representative samples of NOX1, $\alpha$-SMA and FSP-1 are presented. Sections were counterstained with DAPI. Secondary antibody only controls are presented: Anti-rabbit (cat. no. ab6717; Abcam) for NOX1, anti-mouse (cat. no. A32727; Thermo Fisher Scientific, Inc.) for $\alpha$-SMA or FSP-1. Arrows and insets indicate positive staining in fibroblastic cells. NOX1 overlapped with both $\alpha$-SMA and FSP-1-expressing cells, and co-localized with the positive stroma of $\alpha$-SMA and FSP-1, thereby indicating that NOX1 was positively expressed in both $\alpha$-SMA and FSP-1 positive fibroblasts in the stroma of GBCs. NOX1, nicotinamide adenine dinucleotide phosphate oxidase 1; GBCs, gallbladder cancers; $\alpha$-SMA, $\alpha$-smooth muscle actin; FSP-1, fibroblast secreted protein-1.

classification of gene numbers such as biological processes (Fig. 3B). A total of 466 upregulated genes $(\mathrm{FC}>1.5)$ and 596 downregulated genes $(\mathrm{FC}<0.67)$ were identified in GCAFs/NFs according to the inclusion criteria, and of the total 466 upregulated genes, the NOX1 gene was significantly upregulated $(\mathrm{FC}=2.49)$ in GCAFs (Fig. 3C). Furthermore, the expression of NOX1 mRNA was significantly increased in all of the GCAFs compared with adjacent gallbladder NFs (mean \pm SD, $11.45 \pm 1.03 ; 7.04 \pm 0.32 ; 17.58 \pm 0.74$, all $\mathrm{P}<0.05$; Fig. 4). The results were consistent with the results of the
Affymetrix chip analysis. Thus, the results revealed that NOX1 expression was upregulated in GCAFs.

NOX1 expression is upregulated in co-cultures of GBC-SD cells/GCAFs. To further verify the upregulated NOX1 expression in GCAFs, the present study detected the expression of NOX1 at the protein level in the simulated structures (spheroid formation) of human GBC and adjacent tissues using the co-cultures of GBC-SD cells/GCAFs or NFs by IHC and western blotting in vitro. Co-culture of GBC-SD cells with 
Table II. Relationship between the expression of NOX1 in the stroma of GBC and clinicopathological parameters in patients with GBCs.

\begin{tabular}{|c|c|c|c|c|c|}
\hline \multirow[b]{2}{*}{ Variable } & \multirow[b]{2}{*}{$\mathrm{n}$} & \multicolumn{2}{|c|}{ NOX-1 expression [n (\%)] } & \multirow[b]{2}{*}{$\chi^{2}$ value } & \multirow[b]{2}{*}{ P-value } \\
\hline & & Low & High & & \\
\hline \multicolumn{6}{|l|}{ Sex } \\
\hline Male & 25 & $14(56)$ & $11(44)$ & \multirow[t]{2}{*}{0.006} & \multirow[t]{2}{*}{0.937} \\
\hline Female & 40 & $22(55)$ & $18(45)$ & & \\
\hline \multicolumn{6}{|l|}{ Age (years) } \\
\hline$>65$ & 34 & $21(61.8)$ & $13(38.2)$ & \multirow[t]{2}{*}{1.174} & \multirow[t]{2}{*}{0.279} \\
\hline$\leq 65$ & 31 & $15(48.4)$ & $16(51.6)$ & & \\
\hline \multicolumn{6}{|l|}{ Tumor size $(\mathrm{cm})$} \\
\hline$>3$ & 27 & $14(51.9)$ & $13(48.1)$ & \multirow[t]{2}{*}{0.233} & \multirow[t]{2}{*}{0.629} \\
\hline$\leq 3$ & 38 & $22(57.9)$ & $16(42.1)$ & & \\
\hline \multicolumn{6}{|l|}{ Tumor location } \\
\hline Bottom & 26 & $18(69.2)$ & $8(30.8)$ & \multirow[t]{2}{*}{3.362} & \multirow[t]{2}{*}{0.067} \\
\hline Corporis and others & 39 & $18(46.2)$ & $21(53.8)$ & & \\
\hline \multicolumn{6}{|l|}{ Histological type } \\
\hline Adenocarcinoma & 61 & $34(55.7)$ & $27(44.3)$ & \multirow[t]{2}{*}{0.000} & \multirow[t]{2}{*}{1.000} \\
\hline Others ${ }^{\mathrm{a}}$ & 4 & $2(50)$ & $2(50)$ & & \\
\hline \multicolumn{6}{|l|}{ Differentiation degree } \\
\hline G1 (high) & 11 & $8(72.7)$ & $3(27.3)$ & \multirow[t]{3}{*}{6.346} & \multirow[t]{3}{*}{$0.042^{\mathrm{b}}$} \\
\hline G2 (moderate) & 29 & $19(65.5)$ & $10(34.5)$ & & \\
\hline G3 (poor) & 25 & $9(36)$ & $16(64)$ & & \\
\hline \multicolumn{6}{|l|}{ Nevin stage } \\
\hline S1-S2 & 8 & 7 (87.5) & $1(12.5)$ & \multirow[t]{2}{*}{2.470} & \multirow[t]{2}{*}{0.116} \\
\hline S3-S5 & 57 & $29(50.9)$ & $28(49.1)$ & & \\
\hline \multicolumn{6}{|l|}{ Lymph node metastasis } \\
\hline$(-)$ & 44 & $21(47.7)$ & $23(52.3)$ & \multirow[t]{2}{*}{3.232} & 0.072 \\
\hline$(+)$ & 21 & $15(71.4)$ & $6(28.6)$ & & \\
\hline Liver infiltration & & & & & \\
\hline$(+)$ & 31 & $15(48.4)$ & $16(51.6)$ & 1.174 & 0.279 \\
\hline$(-)$ & 34 & $21(61.8)$ & $13(38.2)$ & & \\
\hline Venous invasion & & & & & \\
\hline$(+)$ & 29 & $12(41.4)$ & $17(58.6)$ & 4.156 & $0.041^{\mathrm{b}}$ \\
\hline$(-)$ & 36 & $24(66.7)$ & $12(33.3)$ & & \\
\hline Curability & & & & & \\
\hline R0 & 32 & $24(75)$ & $8(25)$ & 9.815 & $0.002^{\mathrm{b}}$ \\
\hline $\mathrm{R} 1, \mathrm{R} 2$ & 33 & $12(36.4)$ & $21(63.6)$ & & \\
\hline
\end{tabular}

${ }^{a}$ Mucinous adenocarcinoma, squamous cell carcinoma, adenosquamous carcinoma; ${ }^{\mathrm{b}} \mathrm{P}<0.05$, statistically significant. NOX1, nicotinamide adenine dinucleotide phosphate oxidase 1; GBC, gallbladder cancer.

GCAFs strongly enhanced the ability to form spheroids with higher viability; while GBC-SD cells co-cultured with NFs formed loose cell aggregates on day 7. Notably, as determined by IHC, the expression of NOX1 protein was significantly upregulated in the GBC-SD+GCAFs co-culture when compared with GBC-SD+NFs co-culture in vitro $(0.668 \pm 0.058$ vs. $0.465 \pm 0.045 \%$; $\mathrm{P}<0.01$; Fig. $5 \mathrm{~A}$ ); using western blotting, the expression of NOX1 protein in the GBC-SD+GCAFs co-culture was also significantly upregulated compared with the GBC-SD+NFs co-culture in vitro $(0.715 \pm 0.077$ vs. $0.318 \pm 0.031$, relative gray value; $\mathrm{P}<0.001$; Fig. 5B). Thus, the present study further verified that NOX1 expression was upregulated in GCAFs.

\section{Discussion}

NOX1, as one of the members of the NADPH oxidase family, is thought to play a vital role in tumorigenesis and tumor 

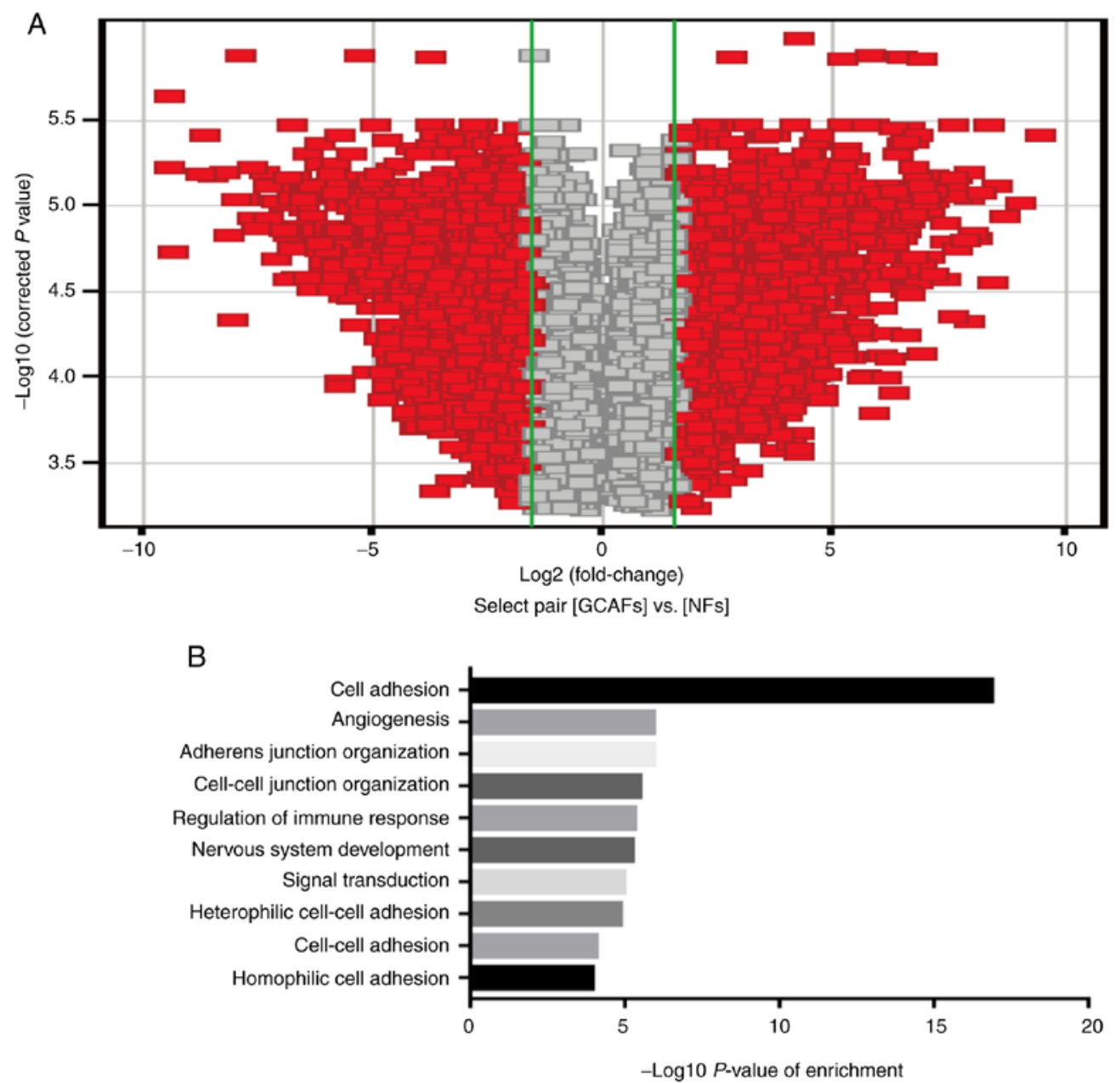

GO analysis for upregulated genes in GCAFs vs. NFs

C

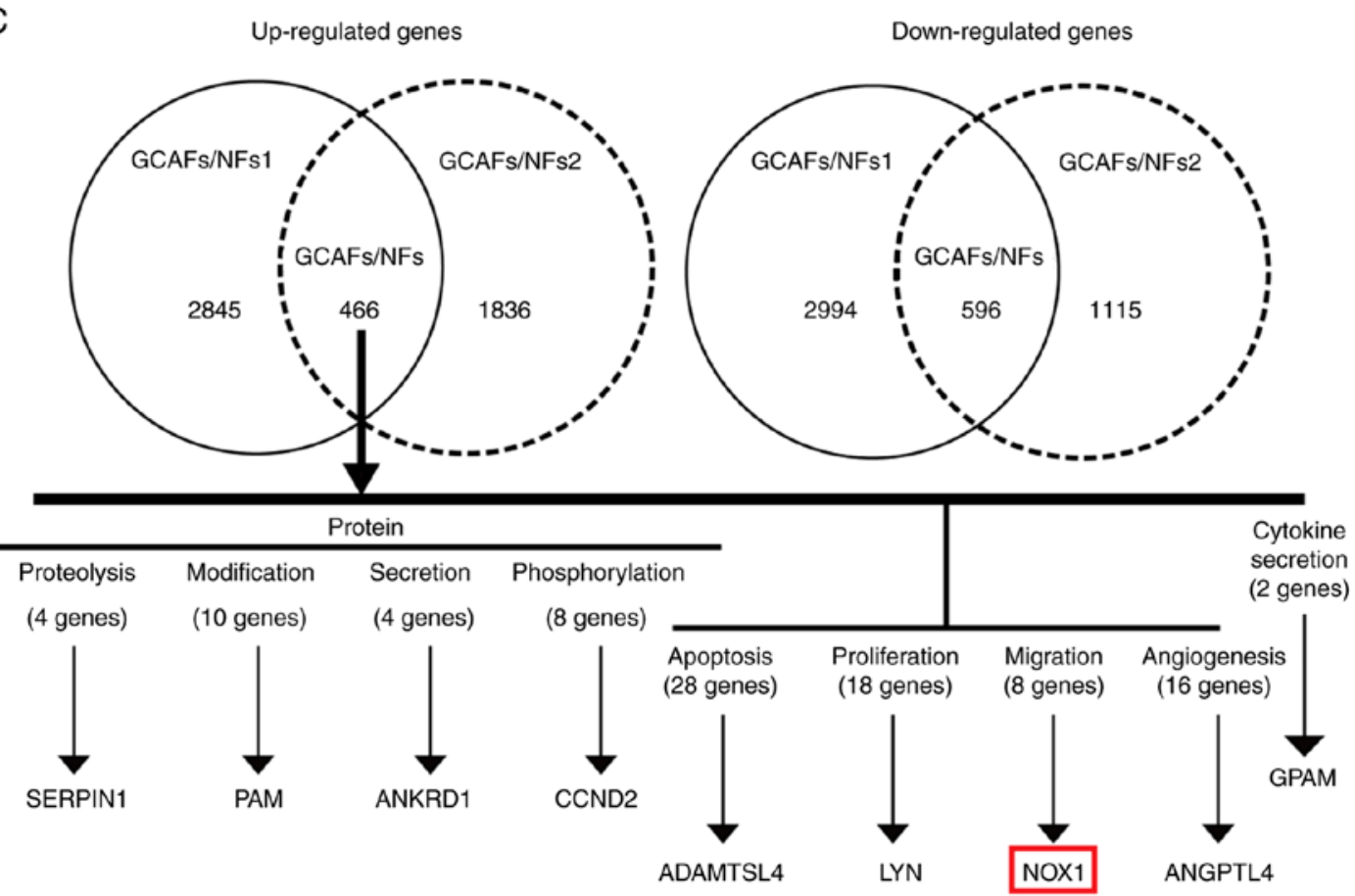

Figure 3. Identification of upregulated NOX1 expression in GCAFs in vitro. (A) Affymetrix chip analysis of the gene expression profile for GCAFs and NFs using the Affymetrix GeneChip Human 1.0ST array. Volcano-map depicting significantly affected genes in GCAFs and NFs. (B) GO analysis revealed the genes with upregulated expression, based on the classification of gene numbers such as biological processes. (C) A total of 466 upregulated genes (FC >1.5) and 596 downregulated genes $(\mathrm{FC}<0.67$ ) were identified in GCAFs/NFs according to the inclusion criteria, and the NOX1 gene was significantly upregulated (FC=2.49) in GCAFs. NOX1, nicotinamide adenine dinucleotide phosphate oxidase 1; GCAFs, gallbladder cancer associated fibroblasts; NFs, normal fibroblasts; GO, Gene Ontology; FC, fold change. 
Table III. Univariate and multivariate analysis of overall survival rate of GBC patients with Cox proportional hazards model.

\begin{tabular}{|c|c|c|c|c|c|c|}
\hline \multirow[b]{2}{*}{ Variable } & \multicolumn{3}{|c|}{ Univariate analysis } & \multicolumn{3}{|c|}{ Multivariate analysis } \\
\hline & HR & $95 \% \mathrm{CI}$ & P-value & HR & $95 \% \mathrm{CI}$ & P-value \\
\hline \multicolumn{7}{|l|}{ Sex } \\
\hline Male vs. female & 1.283 & $0.748-2.203$ & 0.365 & & & \\
\hline \multicolumn{7}{|l|}{ Age (years) } \\
\hline$>65$ vs. $\leq 65$ & 1.109 & $0.652-1.888$ & 0.703 & & & \\
\hline \multicolumn{7}{|l|}{ Tumor size $(\mathrm{cm})$} \\
\hline$>3.0$ vs. $\leq 3.0$ & 1.425 & $0.833-2.436$ & 0.196 & & & \\
\hline \multicolumn{7}{|l|}{ Tumor location } \\
\hline Bottom vs. corporis and other & 0.771 & $0.447-1.331$ & 0.351 & & & \\
\hline \multicolumn{7}{|l|}{ Histological type } \\
\hline Adenocarcinoma vs. Other ${ }^{\mathrm{a}}$ & 0.250 & $0.086-0.727$ & $0.011^{\mathrm{b}}$ & 0.308 & 0.105-0.902 & $0.032^{\mathrm{b}}$ \\
\hline \multicolumn{7}{|l|}{ Differentiation degree } \\
\hline G1 vs. G2 and G3 & 0.263 & $0.111-0.622$ & $0.002^{\mathrm{b}}$ & 0.038 & $0.151-0.974$ & $0.044^{\mathrm{b}}$ \\
\hline \multicolumn{7}{|l|}{ Nevin staging } \\
\hline S3-S5 vs. S1-S2 & 3.732 & $1.339-10.401$ & $0.012^{\mathrm{b}}$ & & & \\
\hline \multicolumn{7}{|l|}{ Lymph node metastasis } \\
\hline \multicolumn{7}{|l|}{ Liver infiltration } \\
\hline $\begin{array}{l}\text { Venous invasion } \\
(+) \text { vs. (-) }\end{array}$ & 2.771 & $1.615-4.756$ & $\mathrm{P}<0.001^{\mathrm{b}}$ & 2.375 & $1.363-4.139$ & $0.002^{\mathrm{b}}$ \\
\hline $\begin{array}{l}\text { Curability } \\
\text { R1, R2 vs. R0 }\end{array}$ & 2.903 & $1.672-5.041$ & $\mathrm{P}<0.001^{\mathrm{b}}$ & 1.833 & $1.010-3.325$ & $0.046^{\mathrm{b}}$ \\
\hline \multicolumn{7}{|l|}{ NOX1 expression in GBC stroma } \\
\hline
\end{tabular}

${ }^{a}$ Mucinous adenocarcinoma, squamous cell carcinoma, adenosquamous carcinoma. ${ }^{b} \mathrm{P}<0.05$, statistically significant. GBC, gallbladder cancer; NOX1, nicotinamide adenine dinucleotide phosphate oxidase 1; HR, Hazard ratio; CI, confidence interval.

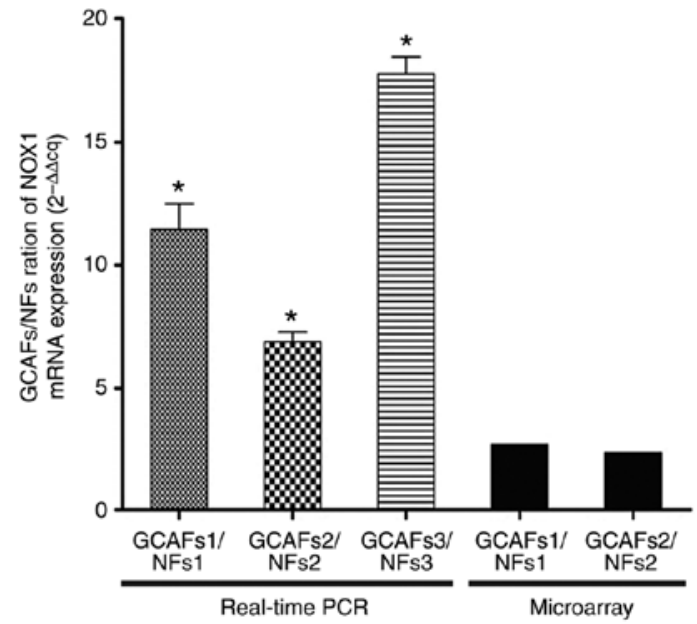

Figure 4. Expression of the NOX1 gene at the mRNA level was detected via reverse transcription-quantitative PCR. The expression of NOX1 mRNA was significantly increased in all of the GCAF groups when compared with the adjacent gallbladder NFs $\left(11.45 \pm 1.03 ; 7.04 \pm 0.32 ; 17.58 \pm 0.74,{ }^{*} \mathrm{P}<0.05\right.$, GCAFs vs. NFs), which was consistent with the results of Affymetrix chip analysis. Therefore, NOX1 expression was upregulated in GCAFs. NOX1, nicotinamide adenine dinucleotide phosphate oxidase 1; GCAFs, gallbladder cancer associated fibroblasts; NFs, normal fibroblasts. development through the generation of ROS and important intracellular signaling molecules $(27,28)$, has been reported to be highly expressed in a variety of tumor types such as gastric (29-31) and liver cancers (32), and is associated with the poor prognosis of these patients. However, these studies mainly focused on the expression of NOX1 in the tumor cells themselves. Recently, some studies have investigated the effect of NOX1 expression in fibroblasts on tumorigenesis and the development of the tumor. Increased NOX1 expression in fibroblasts has the ability to induce malignant transformation and can produce tumors in athymic mice (37). NOX1 converted tumors from dormant to aggressive growth, by rendering them capable of forming well vascularized tumors and inducing molecular markers of angiogenesis, and revealed that NOX1 is a potent trigger of the angiogenic switch (38). Fibroblasts regulated ROS production via NOX1 and NOX4 to mediate cytokine-triggered DNA damage, which may contribute to malignant transformation (39). However, the expression of NOX1 in tumor stroma, particularly GCAFs, and its role in tumor prognosis have not been well clarified. In the present study, it was firstly observed that NOX1 expression was upregulated in the stroma of GBCs compared with GBBLs and 
A
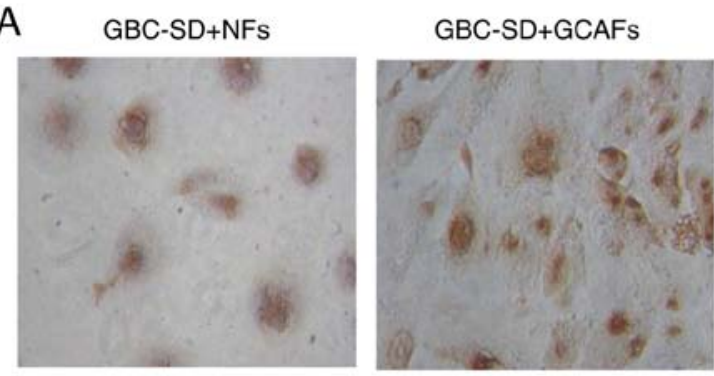

B

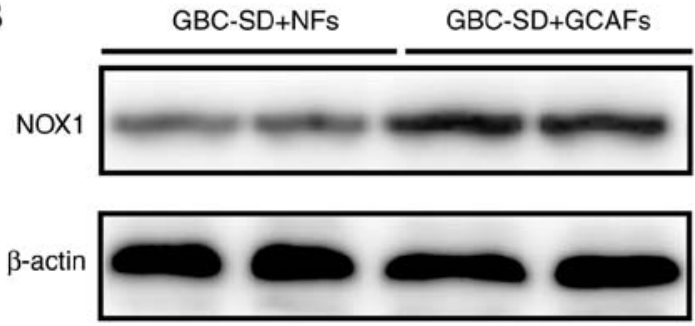

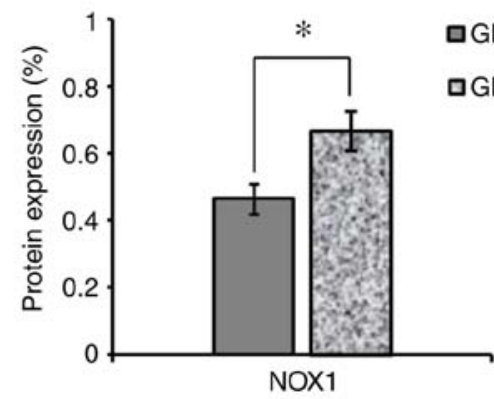

口GBC-SD+NFs

口GBC-SD+GCAFs

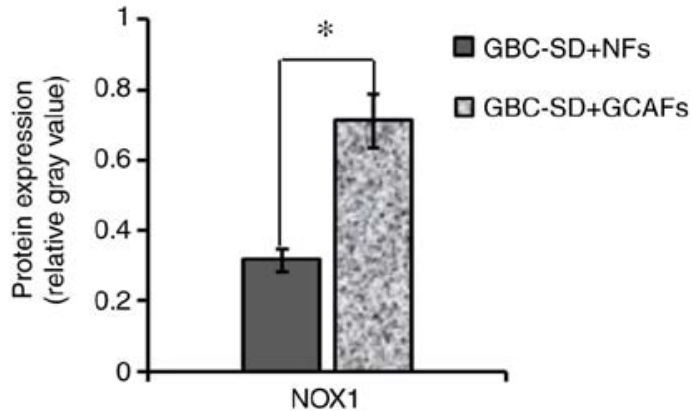

Figure 5. Expression of NOX1 protein in the co-cultures of GBC-SD and GCAFs or NFs in vitro. (A) Immunohistochemistry (magnification, $\mathrm{x} 200$ ): The expression of NOX1 protein was significantly upregulated in the GBC-SD+GCAFs co-culture compared with the GBC-SD+NFs co-culture in vitro. ${ }^{*} \mathrm{P}<0.01$. (B) Western blot analysis: The expression of NOX1 protein in the GBC-SD+GCAFs co-culture was also significantly upregulated compared with the GBC-SD+NFs co-culture in vitro. ${ }^{\mathrm{P}}<0.001$. NOX1, nicotinamide adenine dinucleotide phosphate oxidase 1; GCAFs, gallbladder cancer associated fibroblasts; NFs, normal fibroblasts; GBC, gallbladder cancer.

GBPLs, and the expression of NOX1 in the stroma of GBCs was localized to stromal fibroblasts expressing $\alpha$-SMA and FSP-1 using CIF staining. Secondly, the results revealed that NOX1 expression was upregulated in GCAFs in vitro using Affymetrix chip analysis of the gene expression profile and RT-qPCR analysis for GCAFs/NFs. Finally, it was identified that NOX1 was highly expressed in the simulated spheroid formation of the GBC-SD+GCAFs co-culture, compared with the GBC-SD+NFs co-culture, using IHC and western blotting. Thus, it is believed that NOX1 expression was upregulated in GCAFs.

An increasing body of evidence has revealed that the TME serves a critical role in the growth and development of the tumor. The TME, the 'soil' of tumor growth, is composed of cancer cells and various interstitial cells, including the extracellular matrix, proteolytic enzymes, cytokines and chemokines, and interacts with tumors by altering the proteome and degradome (8-10). As a prominent member of the stroma and as the most stromal or interstitial cells in the TME, CAFs have their own gene expression profiles that are different from NFs, and interact with cancer cells via a variety of signals to affect the TME, tumorigenesis, development and therapeutic tolerance of cancer cells (11-13), which are also associated with the prognosis of various tumors by expressing FAP, FGF-2, IL-1 $\beta$-IRAK4 and Podoplanin (15,16,40-44). Notably, the presence of reactive stroma in the TME, particularly in CAFs, was revealed to affect the tumor treatment effectiveness and was associated with tumor drug resistance (17-20). Anti-CAFs can effectively prevent tumor progression before tumor invasion, and prolong the survival of patients compared with chemotherapy alone in pancreatic and other cancer treatments (20-23). In the present study, NOX1 expression was significantly upregulated in the stroma of GBC tissues with positive expression of $\alpha$-SMA and FSP-1 in vivo, and NOX1 was highly expressed in GCAFs and the simulated spheroid formation of the GBC-SD+GCAFs co-culture with positive expression of $\alpha$-SMA and FAP, compared with NFs with negative expression of $\alpha$-SMA and FAP in vitro. It is recognized that $\alpha$-SMA, FSP-1 and FAP are the most important stromal or interstitial markers, their positive expression localized to stromal GCAFs. Thus, the present study verified that NOX1 expression was upregulated in GCAFs. To determine the significance of upregulated NOX1 expression in GCAF, the present study further analyzed the relationship between NOX1 expression and the clinicopathological characteristics and prognostic factors of GBC patients. The results revealed the upregulated NOX1 expression in the stroma of GBCs; GCAFs were correlated with aggressive characteristics such as differentiation degree, venous invasion, resection methods (all $\mathrm{P}<0.05)$, and the lower survival rate $(\mathrm{P}=0.025$, log-rank test $)$ of GBC patients. Stromal NOX1 expression $(\mathrm{P}=0.047)$ was an independent prognostic factor for the OS rate of GBC patients. Thus, it was concluded that GBC patients with upregulated NOX1 expression in GCAFs have a poorer prognosis.

GBC, as a highly malignant tumor, is insensitive to chemotherapy and radiotherapy. It was recently reported that NOX1 overexpression mediated the chemical resistance of cisplatin through elevated intracellular ROS levels by activating the HIF1 $\alpha /$ MDR1 signaling pathway in GBC cells, and that NOX1 was a novel accelerant of chemoresistance in GBC (45). It is possible that high stromal NOX1 expression in GBC after chemotherapy maintains the viability of tumor cells by secreting some anti-drug molecules. NOX1-targeted therapeutics may be exploited as a strategy for increasing the efficacy of cisplatin treatment (45). Collectively, NOX1 expression was upregulated in GCAFs and was associated with the 
unfavorable prognosis of GBC patients, which will enable the establishment of novel biomarkers and potential therapeutic targets that will more accurately predict patient prognosis and responsiveness to treatments for human GBCs.

In conclusion, the present study revealed that NOX1 expression was upregulated in the stroma of GBCs, and that GCAFs with positive $\alpha$-SMA and FSP-1 expression were sources of NOX1 expression. Upregulated NOX1 expression was related to the tumor differentiation degree, venous invasion and survival rate of patients, and appeared to be an independent prognostic factor, thereby suggesting a poor prognosis in patients with GBC. An in-depth study of NOX1 expression in GCAFs and its molecular mechanisms will contribute to the development of novel prognostic markers and therapeutic targets for human GBCs.

\section{Acknowledgements}

Not applicable.

\section{Funding}

The present study was supported by funds from the National Nature Science Foundation of China (grant nos. 30672073 and 81372614) and the Shanghai Science and Technology Commission Research Project (grant no. 19411966300).

\section{Availability of data and materials}

Not applicable.

\section{Authors' contributions}

FTW, MH, KHA and YZF conceived and designed the experiments. FTW, MH, KHA and YZF performed the experiments. FTW, GLX and XPL analyzed the data. GLX and XPL contributed reagents/materials/analysis tools. FTW and YZF wrote the paper. All authors have read and approved the final manuscript and agree to be accountable for all aspects of the research in ensuring that the accuracy or integrity of any part of the work are appropriately investigated and resolved.

\section{Ethics approval and consent to participate}

The present study was approved by the Ethics Committee and the Institutional Review Board of Tongji Hospital. Written informed consent was obtained from all patients.

\section{Patient consent for publication}

Not applicable.

\section{Competing interests}

The authors declare that they have no competing interests.

\section{References}

1. Valle JW, Lamarca A, Goyal L, Barriuso J and Zhu AX: New horizons for precision medicine in biliary tract cancers. Cancer Discov 7: 943-962, 2017.
2. Siegel RL, Miller KD and Jemal A: Cancer Statistics, 2016. CA Cancer J Clin 66: 7-30, 2016

3. Chen W, Zheng R, Baade PD, Zhang S, Zeng H, Bray F, Jemal A Yu XQ and He J: Cancer Statistics in China, 2015. CA Cancer J Clin 66: 115-132, 2016.

4. Liu ZY, Cao J, Zhang JT, Xu GL, Li XP, Wang FT, Ansari KH, Mohamed $\mathrm{H}$ and Fan YZ: Ring finger protein 125, as a potential highly aggressive and unfavorable prognostic biomarker, promotes the invasion and metastasis of human gallbladder cancers via activating the TGF- $31-S M A D 3-I D 1$ signaling pathway. Oncotarget 8: 49897-49914, 2017.

5. Hundal R and Shaffer EA: Gallbladder cancer: Epidemiology and outcome. Clin Epidemiol 6: 99-109, 2014.

6. Horgan AM, Amir E, Walter T and Knox JJ: Adjuvant therapy in the treatment of biliary tract cancer: A systematic review and meta-analysis. J Clin Oncol 30: 1934-1940, 2012.

7. Malka D, Cervera P, Foulon S, Trarbach T, de la Fouchardière C, Boucher E, Fartoux L, Faivre S, Blanc JF, Viret F, et al: Gemcitabine and oxaliplatin with or without cetuximab in advanced biliary-tract cancer (BINGO): A randomised, open-label, non-comparative phase 2 trial. Lancet Oncol 15: 819-828, 2014

8. Liotta L and Kohn E: The microenvironment of the tumour-host interface. Nature 411: 375-379, 2001.

9. Kim JB, Stein R and O'Hare MJ: Tumour-stromal interactions in breast cancer: The role of stroma in tumourigenesis. Tumour Biol 26: 173-185, 2005.

10. Reisfeld R: The tumor microenvironment: A target for combination therapy of breast cancer. Crit Rev Oncog 18: 115-133, 2013.

11. Finak G, Bertos N, Pepin F, Sadekova S, Souleimanova M, Zhao H, Chen H, Omeroglu G, Meterissian S, Omeroglu A, et al: Stromal gene expression predicts clinical outcome in breast cancer. Nat Med 14: 518-527, 2008.

12. Ahn S, Cho J, Sung J, Lee JE, Nam SJ, Kim KM and Cho EY: The prognostic significance of tumor-associated stroma in invasive breast carcinoma. Tumor Biol 33: 1573-1580, 2012.

13. Shi S, Liang C, Xu J, Meng Q, Hua J, Yang X, Ni Q and Yu X: The strain ratio as obtained by endoscopic ultrasonography elastograhy correlates with the stroma proportion and the prognosis of local pancreatic cancer. Ann Surg, 2018

14. Orimo A, Gupta PB,Sgroi DC,Arenzana-Seisdedos F, Delaunay T, Naeem R, Carey VJ, Richardson AL and Weinberg RA: Stromal fibroblasts present in invasive human breast carcinomas promote tumor growth and angiogenesis through elevated SDF-1/CXCL12 secretion. Cell 121: 335-348, 2005.

15. Gao Q, Wang XY, Qiu SJ, Zhou J, Shi YH, Zhang BH and Fan J: Tumor stroma reaction-related gene signature predicts clinical outcome in human hepatocellular carcinoma. Cancer Sci 102: $1522-1531,2011$

16. Herrera M, Herrera A, Domínguez G, Silva J, García V, García JM, Gómez I, Soldevilla B, Muñoz C, Provencio M, et al: Cancer-associated fibroblast and M2 macrophage markers together predict outcome in colorectal cancer patients. Cancer Sci 104: 437-444, 2013

17. Micke $P$ and Ostman A: Tumour-stroma interaction: Cancerassociated fibroblasts as novel targets in anti-cancer therapy? Lung Cancer 45 (Suppl 2): S163-S175, 2004.

18. Johansson AC, Ansell A, Jerhammar F, Lindh MB, Grénman R, Munck-Wikland E, Östman A and Roberg K: Cancer-associated fibroblasts induce matrix metalloproteinase-mediated cetuximab resistance in head and neck squamous cell carcinoma cells. Mol Cancer Res 10: 1158-1168, 2012.

19. Affolter A, Schmidtmann I, Mann WJ and Brieger J: Cancer-associated fibroblasts do not respond to combined irradiation and kinase inhibitor treatment. Oncol Rep 29: 785-790, 2013.

20. Gonda TA, Varro A, Wang TC and Tycko B: Molecular biology of cancer-associated fibroblasts: Can these cells be targeted in anti-cancer therapy? Semin Cell Dev Biol 21: 2-10, 2010.

21. Al-Ansari MM, Hendrayani SF, Tulbah A, Al-Tweigeri T, Shehata AIand Aboussekhra A: p16INK4A represses breast stromal fibroblasts migration/invasion and their VEGF-A-dependent promotion of angiogenesis through Akt inhibition. Neoplasia 14: 1269-1277, 2012.

22. Mertens JC, Fingas CD, Christensen JD, Smoot RL, Bronk SF, Werneburg NW, Gustafson MP, Dietz AB, Roberts LR, Sirica AE, et al: Therapeutic effects of deleting cancer-associated fibroblasts in cholangiocarcinoma. Cancer Res 73: 897-907, 2013. 
23. Olive KP, Jacobetz MA, Davidson CJ, Gopinathan A, McIntyre D, Honess D, Madhu B, Goldgraben MA, Caldwell ME, Allard D, et al: Inhibition of Hedgehog signaling enhances delivery of chemotherapy in a mouse model of pancreatic cancer. Science 324: 1457-1461, 2009.

24. Altenhöfer S, Kleikers PW, Radermacher KA, Scheurer P, Rob Hermans JJ, Schiffers P, Ho H, Wingler K and Schmidt HH: The NOX toolbox: Validating the role of NADPH oxidases in physiology and disease. Cell Mol Life Sci 69: 2327-2343, 2012.

25. Bartosz G: Reactive oxygen species: Destroyers or messengers? Biochem Pharmacol 77: 1303-1315, 2009.

26. Schröder K: Isoform specific functions of Nox protein-derived reactive oxygen species in the vasculature. Curr Opin Pharmacol 10 $122-126,2010$

27. Kamata T: Roles of Nox 1 and other Nox isoforms in cancer development. Cancer Sci 100: 1382-1388, 2009.

28. Roy K, Wu Y, Meitzler JL, Juhasz A, Liu H, Jiang G, Lu J, Antony S and Doroshow JH: NADPH oxidases and cancer. Clin Sci (Lond) 128: 863-875, 2015.

29. You X, Ma M, Hou G, Hu Y and Shi X: Gene expression and prognosis of NOX family members in gastric cancer. Onco Targets Ther 11: 3065-3074, 2018.

30. Augusto AC, Miguel F, Mendonça S, Pedrazzoli J Jr and Gurgueira SA: Oxidative stress expression status associated to Helicobacter pylori virulence in gastric diseases. Clin Biochem 40: 615-622, 2007.

31. Montalvo-JavéEE, Olguín-Martínez M,Hernández-Espinosa DR, Sánchez-Sevilla L,Mendieta-CondadoE, Contreras-Zentella ML, Oñate-Ocaña LF, Escalante-Tatersfield T, Echegaray-Donde A, Ruiz-Molina JM, et al: Role of NADPH oxidases in inducing a selective increase of oxidant stress and cyclin D1 and checkpoint 1 over-expression during progression to human gastric adenocarcinoma. Eur. J Cancer 57: 50-57, 2016.

32. Eun HS, Cho SY, Joo JS, Kang SH, Moon HS, Lee ES, Kim SH and Lee BS: Gene expression of NOX family members and their clinical significance in hepatocellular carcinoma. Sci Rep 7: $11060,2017$.

33. Linder N, Konsti J, Turkki R, Rahtu E, Lundin M, Nordling S, Haglund C, Ahonen T, Pietikäinen M and Lundin J: Identification of tumor epithelium and stroma in tissue microarrays using texture analysis. Diagn Pathol 7: 22, 2012.

34. Lehr HA, van der Loos CM, Teeling P and Gown AM: Complete chromogen separation and analysis in double immunohistochemical stains using Photoshop-based image analysis. J Histochem Cytochem 47: 119-126, 1999.

35. Zou A, Lambert D, Yeh H, Yasukawa K, Behbod F, Fan F and Cheng N: Elevated CXCL1 expression in breast cancer stroma predicts poor prognosis and is inversely associated with expression of TGF- $\beta$ signaling proteins. BMC Cancer 14: 781, 2014.
36. Livak KJ and Schmittgen TD: Analysis of relative gene expression data using real-time quantitative PCR and the 2(-Delta Delta C(T)) method. Methods 25: 402-408, 2001.

37. Suh YA, Arnold RS, Lassegue B, Shi J, Xu X, Sorescu D, Chung AB, Griendling KK and Lambeth JD: Cell transformation by the superoxide-generating oxidase Mox1. Nature 401: 79-82, 1999.

38. Arbiser JL, Petros J, Klafter R, Govindajaran B, McLaughlin ER Brown LF, Cohen C, Moses M, Kilroy S, Arnold RS and Lambeth JD: Reactive oxygen generated by Nox 1 triggers the angiogenic switch. Proc Natl Acad Sci USA 99: 715-720, 2002.

39. Illeperuma RP, Kim DK, Park YJ, Son HK, Kim JY, Kim J, Lee DY, Kim KY, Jung DW, Tilakaratne WM and Kim J: Areca nut exposure increases secretion of tumor-promoting cytokines in gingival fibroblasts that trigger DNA damage in oral keratinocytes. Int J Cancer 137: 2545-2557, 2015.

40. Franco OE, Shaw AK, Strand DW and Hayward SW: Cancer associated fibroblasts in cancer pathogenesis. Semin Cell Dev Biol 21: 33-39, 2010.

41. Pecqueux C, Arslan A, Heller M, Falkenstein M, Kaczorowski A, Tolstov Y, Sültmann H, Grüllich C, Herpel E, Duensing A, et al: FGF-2 is a driving force for chromosomal instability and a stromal factor associated with adverse clinico-pathological features in prostate cancer. Urol Oncol 36: 365.e15-365.e26, 2018.

42. Zhang D, Li L, Jiang H, Li Q, Wang-Gillam A, Yu J, Head R, Liu J, Ruzinova MB and Lim KH: Tumor-stroma IL1 $\beta$-IRAK4 feedforward circuitry drives tumor fibrosis, chemoresistance, and poor prognosis in pancreatic cancer. Cancer Res 78: 1700-1712, 2018.

43. Wikberg ML, Edin S, Lundberg IV, Van Guelpen B, Dahlin AM, Rutegård J, Stenling R, Oberg A and Palmqvist R: High intratumoral expression of fibroblast activation protein (FAP) in colon cancer is associated with poorer patient prognosis. Tumour Biol 34: 1013-1020, 2013.

44. Schoppmann SF, Berghoff A, Dinhof C, Jakesz R, Gnant M, Dubsky P, Jesch B, Heinzl H and Birner P: Podoplanin-expressing cancer-associated fibroblasts are associated with poor prognosis in invasive breast cancer. Breast Cancer Res Treat 134: 237-244, 2012.

45. Zhan M, Wang H, Chen T, Chen W, Yang L, He M,Xu S and Wang J: NOX1 mediates chemoresistance via HIF1 $\alpha /$ MDR1 pathway in gallbladder cancer. Biochem Biophys Res Commun 468: 79-85, 2015. 